\title{
Le travail de reconfiguration nature/culture du féminin
}

\author{
ou le spectre de l'engagement féministe
}

Delphine Masset

\begin{abstract}
[Résumé] Comment une femme peut-elle changer sa condition féminine ? N'y a-t-il pas plusieurs niveaux de mobilisation, plusieurs attitudes à adopter? Dans cet article, je déploierai la palette d'attitudes que peuvent mobiliser les femmes dans leurs combats féministes en proposant de sortir du cadre formel de la distinction essentialisme-constructivisme. J'analyserai, par ailleurs, le schisme ou la rupture qui s'opère parfois lorsqu'une femme s'engage dans une lutte institutionnelle et pour sa reconnaissance identitaire. Je développerai, enfin, l'idée de « bricolage » du féminin et des luttes féministes dans le but d'une réconciliation de cette rupture.

Mots-clés : féminin, désir, socialisation.
\end{abstract}

Les précédentes auteures ont largement développé les différences entre essentialisme et constructivisme pour appréhender « le féminin ». Pour rappel, selon l'essentialisme, la femme est par nature différente à l'homme et ce, sur le plan physique et psychique. Pour les constructivistes, « la femme » est une construction sociale. Rien de « la femme» n'est intrinsèque si ce n'est ses déterminations biologiques.

Ces distinctions, si elles permettent de fixer les termes du débat, peuvent cependant se révéler dogmatiques et stériles. Quand il s'agit de saisir la manière dont une femme se vit au quotidien ou ce qui est au fondement de la construction de ses désirs, ces distinctions peuvent en effet en compliquer la lecture.

Dans la sphère du vécu, inné et acquis s'entrecroisent, nature et culture se font plus indistinctes... puisque « les dispositifs de pouvoir ne se contentent plus d'être normalisants, ils tendent à être constituants ${ }^{2}$ »(de la sexualité ou de l'identité). Les désirs des femmes, tout en étant un objet de culture, sont effectivement aussi ce qui constitue le plus intimement leur nature.

La marge de manœuvre de l'émancipation féminine se situe sur la crête qui sépare nature et culture. Heureusement, celle-ci ne semble pas impraticable. Comme le dit Judith Butler « on peut certainement concéder que le désir est radicalement conditionné sans affirmer qu'il est radicalement déterminé ${ }^{3} »$. Il y a donc de l'espace entre conditionnement et détermination.

1 Et ce, même depuis l'existence de mouvements féministes. En effet, « montrer que les catégories sont relatives n'implique pas qu'on puisse les faire disparaître (Ecorev, Est-il possible de s'inventer?) ». Autrement dit, identifier l'existence d'une construction sociale de la féminité n'induit pas une disparition des mécanismes de domination et de reproduction sociale à l'égard des femmes.

${ }^{2}$ Gilles Deleuze, «Désir et plaisir », Magazine Littéraire n 325, octobre 1994.

${ }^{3}$ Judith Butler, Défaire le genre, traduction de Maxime Cervulle, Paris : Éditions Amsterdam, 2006, p.64. 
C'est cet interstice qui permettra à chaque femme d'élaborer son propre positionnement et ce, à partir d'une large palette d'attitudes envisageables à l'égard du genre. Il existe en effet une pluralité de modes d'engagement possibles pour prendre part au monde en tant que femme. Un large spectre dont les femmes disposent pour se réinventer.

Ainsi, au travers de cet article, je proposerai de sortir de l'impasse qu'impose la perspective strictement constructiviste du point de vue de la construction identitaire genrée. En concevant la part « culturelle » de la femme comme étant « naturelle », je suggérerai une lecture du vécu féminin qui complexifie son rapport à la "domination ». Souhaitant développer une sociologie du quotidien, je proposerais d'aller voir le bricolage et les agencements dont les femmes peuvent faire preuve pour élaborer leur rapport au genre. En effet, le constructivisme, qui vise à déduire des attitudes féminines des comportements culturels, envisage peu le fait que les femmes (tout comme les hommes) fassent de leur culture leur nature (autrement dit, qu'elle incorpore leur socialisation). Or, qu'elle le veuille ou non, la femme est récipiendaire d'un héritage incarné.

\section{Les divers agencements dans le rapport au genre}

À l'instar des trois courants féministes, il existe trois modes d'agencements mobilisables par les femmes à l'égard de leur genre. Trois manières de jouer à la charnière du rapport nature-culture, trois façons de marcher sur la crête qui les sépare.

(Option 1) La posture " libérale-égalitariste» ou universaliste qui vise l'égalité des genres. Cette posture est propre au mouvement féministe de la première vague. Ce mouvement « insiste avant tout sur la dimension politique des revendications féministes, à savoir les droits civiques. La femme doit être l'égale de l'homme ${ }^{4}$ ». Au travers de ce courant, il s'agira, pour la femme, de transformer les conditions et les institutions sociales qui reproduisent les processus de domination masculine. Cette perspective est constructiviste. Elle visera aussi bien souvent à écarter les clichés féminins de la sphère publique pour que la femme ne doive plus correspondre à un certain genre féminin.

(Option 2) La posture différentialiste. « Ce courant insiste sur la différence naturelle qui existerait entre les hommes et les femmes. Pour ces féministes, les femmes doivent revendiquer la reconnaissance de leur spécificité ${ }^{5}$ ». C’est la perspective essentialiste, proche des mouvements de la reconnaissance, qui visera à retourner le stigmate pour revaloriser l'identité disqualifiée.

(Option 3) La posture « Queer » qui viserait à transcender le genre afin de rendre possible la transversalité des genres. « La théorie queer critique la thèse de l’identité fémi-

\footnotetext{
4 IRESMO, Histoire, théories et actualité du mouvement féministe. Décembre 2011. Page consultable sur : http://iresmo.jimdo.com/2011/12/11/histoire-th\%C3\%A9ories-et-actualit\%C3\%A9-du-mouvement-f\%C3\%A9ministe/ ${ }^{5}$ Idem
} 
nine du courant différentialiste. En distinguant le sexe biologique et le genre, construction sociale, les théoriciennes du queer défendent la thèse selon laquelle les identités ne sont pas naturelles, mais sont des constructions sociales qui peuvent être déconstruites par les individus, en les jouant dans des « performances ». Dans ce cas, il n'y a plus de jugement de valeur porté sur les comportements : il n’y a plus de genre. La femme (et l'homme) peuvent être masculin(e)s et féminin(e)s. Le mouvement Queer vise en effet l'abolition des frontières identitaires', la non-imposition d'une identité sexuée (voir à ce sujet Monique Wittig et la pensée « straight») et donc la fin du « sexe». C'est la libération des identités.

Selon que la perspective adoptée soit constructiviste ou essentialiste, la femme aura donc le choix entre militer pour une égalité de droits et rejeter publiquement les clichés de la femme ou requalifier publiquement l'identité féminine. La conjonction de l'essentialisme et du constructivisme se retrouvant dans la doctrine «Queer » qui vise à jouer la pluralité et à faire sauter l'idée de genre.

Mais à ces différentes luttes publiques et politiques font échos autant de luttes individuelles et identitaires. Les femmes sont en effet confrontées à la question du genre au niveau le plus élémentaire de leur identité : leurs désirs, leurs émotions, leurs réflexes, tout cela est fonction, pour partie, de schémas incorporés. En effet, la « domination masculine ${ }^{7}$ » va jusqu'à modeler la construction identitaire féminine. Malgré les luttes féministes et revendications d'en finir avec les clichés féminins, l'incorporation des normes et des comportements ne s'est, elle, évidemment pas dissipée. L'individualité reste aussi plastique aux effets de culture. Il n'y a pas de femme (comme il n'y a pas d'homme) non sujette aux imaginaires sociaux qui aurait élevé solitairement ses propres normes, repères. Il n'y a donc que peu de femmes sans une culture de la «féminité » incorporée.

Les luttes identitaires et individuelles sont cependant bien souvent le reflet inversé des luttes politiques. Le travail sur une « culture » machiste inadaptée ignorera l'existence d'un « naturel féminin ». Et inversement : un " accomplissement de sa féminité » laissera en friche tout le travail culturel d'un changement de société. La femme se verra donc bien souvent obligée de choisir entre une identité opprimée accompagnée d'une féminité accomplie ou d'une identité affranchie (par le combat social) et d'une féminité mutilée. Comme l'énonce Simone de Beauvoir :

«Par l'association du phallus et de la transcendance, il se trouve que ses [l'homme] réussites sociales ou spirituelles le dotent d'un prestige viril. II n'est pas divisé. Tandis qu'il est demandé à la femme pour accomplir sa féminité de

6Éric Macé, « Le piège de la "cause des femmes" (Troisième partie) », novembre 2003.

Voir : http://Imsi.net

${ }^{7}$ Qui est une domination commune : celle, généralisée, de l'incorporation des normes par les institutions sociales que sont la famille, l'école, la profession etc. 
se faire objet et proie, c'est-à-dire de renoncer à ses revendications de sujet souverain. C'est ce conflit qui caractérise singulièrement la situation de la femme affranchie. Elle refuse de se cantonner dans son rôle de femelle parce qu'elle ne veut pas se mutiler; mais ce serait aussi une mutilation de répudier son sexe ${ }^{8}$.»

Ainsi, comme le dit Simone de Beauvoir, que la femme se réconcilie avec son être social ou qu'elle tente de s'affranchir de celui-ci, celle-ci connaîtra une tension irrésolue soit au niveau social, soit au niveau individuel. Plus schématiquement, on pourrait dire que

\begin{tabular}{|l|l|l|l|}
\hline Rapport à la... & «CULTURE » & « NATURE » & \\
\hline Constructivisme & Identité affranchie & Féminité mutilée & $\begin{array}{l}\text { Mouvement social } \\
\text { pour la condition } \\
\text { de la femme, muti- } \\
\text { lation de sa fémi- } \\
\text { nité. }\end{array}$ \\
\hline Essentialisme & Identité opprimée & $\begin{array}{l}\text { Féminité } \\
\text { "accomplie » }\end{array}$ & $\begin{array}{l}\text { Mutilation sociale, } \\
\text { reconnaissance de } \\
\text { sa féminité. }\end{array}$ \\
\hline
\end{tabular}

\section{Le spectre de l'engagement féminin, conflit intérieur ou conflit social ?}

Selon la manière dont la femme arbitrera son rapport nature/culture dans son quotidien, la femme pourra se vivre affranchie et mutilée, ou accomplie au prix d'un renoncement de sa souveraineté. En effet, chez chaque personne qui adopte (par la reproduction de comportements, la force de persuasion de certaines images sociales, etc.) une série de comportements sociaux disqualifiés, il y a un schisme possible : celui qui désunit les comportements volontairement adoptés et les comportements inconsciemment reproduits.

\subsection{Au niveau de la sphère privée}

Soit la femme arrive à redonner de la valeur à ses comportements disqualifiés socialement (et arrive à dépasser la dissonance cognitive) en reléguant les auteurs de cette hiérarchisation (choix essentialisme), soit elle aspire à développer d'autres comportements ainsi qu'à éprouver d'autres sensations jugeant que ceux-ci ne proviennent pas de son libre arbitre, et entre alors en conflit avec une partie d'elle-même (choix constructiviste).

${ }^{8}$ DE BEAuvolR Simone, Le Deuxième Sexe, tome II, Paris : Gallimard, 1976, pp. 590-591. 
Dans le « choix constructiviste » d'une femme qui s'engage dans une lutte contre ses propres réflexes, chaque action non conforme à une égalité homme-femme la séparerait un peu plus d'elle-même. Ainsi, la position constructiviste radicale inclinerait plutôt les esprits féminins à rejeter les parties d'elle-même qui auraient été acculturées à la culture patriarcale.

A contrario, en n'associant pas la reproduction des comportements genrés (la gentillesse, le don de soi, la compassion, etc.) à un échec (choix essentialiste), la femme évite bien des tensions et a le privilège de connaître une forme de réconciliation.

\subsection{Au niveau de la sphère publique}

Mais si la posture essentialiste permet aux femmes de vivre en harmonie avec ellesmêmes (avec les différentes parties d'elles-mêmes, et de cohabiter avec leur libre arbitre tout autant que leurs déterminismes), les tenants du constructivisme leur reprocheront de ne plus percevoir l'intérêt d'une lutte sociale, au niveau de la société. C'est l'effet miroir de la construction identitaire vis-à-vis de la société : ce qui est harmonieux en soi sera aisément imaginé harmonieux dans la société.

Le cas contraire d'une tentative de transformations des parties de soi indisciplinées à l'égalité des genres débouchera, lui, sur le maintien d'une lutte sociale. En effet, ces sentiments motiveront l'envie que soit reconnu et requalifié, au niveau de la société, cette identité. En ce sens, ce que ces femmes ne voudraient plus voir de disqualifié en elles-mêmes, elles devraient le dissoudre dans la société.

Ce dilemme n'est pas propre à la femme. Il appartient à toutes les personnes socialisées suffisamment tôt dans un univers qui les a disqualifiés. Pour peu qu'il y ait eu incorporation (des compétences... jusqu'aux sensations) d'un rôle " de seconde zone », il devient difficile pour un individu de se libérer des structurations sociales mais aussi de son inconscient (puisqu'elles ont " pris corps ») sans nourrir un dégoût vis-à-vis de lui-même de par le rejet qu'il nourrirait pour des comportements reproduits malgré lui.

Si l'on reprend notre triptyque, quelles sont les perspectives émancipatrices qu'apportent ces courants, par rapport à la dimension identitaire du genre ? Du point de vue de la construction de l'identité et des agencements de celle-ci dans la sphère privée, quelles sont les grandes possibilités de changement dans lesquelles la femme peut s'engager?

Un déroulé symétrique aux mouvements sociaux féministes peut en réalité être proposé au niveau de la posture individuelle envisageable à adopter : 


\begin{tabular}{|l|l|l|}
\hline & Mouvements sociaux & Posture individuelle \\
\hline $\begin{array}{l}\text { Queer. Vise l'abolition des } \\
\text { frontières identitaires }\end{array}$ & $\begin{array}{l}\text { Revendiquer le dépassement } \\
\text { et le mélange des genres }\end{array}$ & $\begin{array}{l}\text { Mélanger ou dépas- } \\
\text { ser les genres }\end{array}$ \\
\hline $\begin{array}{l}\text { Constructivisme. Vise } \\
\text { l'égalité des conditions } \\
\text { sociales homme-femme }\end{array}$ & $\begin{array}{l}\text { Rejeter publiquement les cli- } \\
\text { chés de la femme, militer } \\
\text { pour une égalité de droits }\end{array}$ & $\begin{array}{l}\text { Rejeter / disqualifier } \\
\text { chez soi une série de } \\
\text { comportements gen- } \\
\text { rés }\end{array}$ \\
\hline $\begin{array}{l}\text { Essentialisme. Vise la re- } \\
\text { connaissance de l'identité }\end{array}$ & $\begin{array}{l}\text { Requalifier publiquement } \\
\text { l'identité féminine }\end{array}$ & $\begin{array}{l}\text { Requalifier son iden- } \\
\text { tité féminine, se ré- } \\
\text { concilier avec son } \\
\text { identité féminine }\end{array}$ \\
\hline
\end{tabular}

Dans le premier cas, cette femme mobilise la doctrine Queer dans son quotidien. Le mélange des genres que permet le mouvement Queer semble en effet idéal en ce qu'il arrive à faire exploser les canons, aussi bien dans la sphère publique que dans la sphère privée. Sa pratique restera cependant en partie inaccessible à toute une série de femmes. Comme nous le suggère Ghaliya Djelloul dans son article, la capacité des femmes à politiser leur identité genrée reste inégalement répartie. Les femmes détenant moins de capitaux socio-économiques auront moins de ressources et d'opportunités pour s'ériger contre l'ordre établi et moduler leurs rapports au genre. En effet, les modèles alternatifs et argumentatifs risquent bien souvent de manquer aux femmes qui ne sont pas proches des mouvements féministes. De nombreuses femmes n'ont en effet jamais eu l'occasion de se confronter aux réflexions « troisième vague » du mouvement féministe et de ce fait, développeront probablement peu un rapport transgenre à elles-mêmes.

Dans le cas suivant, elle adopte une posture constructiviste. Elle résistera alors à ses propres réflexes genrés, au prix d'une violence exercée à son égard. L’option constructiviste l'amènera en effet à probablement s'insurger contre l'incorporation des comportements genrés. Le risque entretenu par cette position est qu'elle se retrouve dans l'impossibilité de neutraliser son individualité genrée. L’individu post-genre (et non pas transgenre comme la doctrine Queer) auquel aspirent les féministes constructivistes reste en effet une pure visée. Dépasser le genre, au niveau de la psyché et des mécanismes inconscients, s'avère périlleux, voire, impossible. Si une femme peut s'imaginer « autre » dans une société qui n'aurait pas véhiculé une série de stéréotypes, elle ne peut se vivre totalement libérée de ces stéréotypes. Encore une fois : ce que l'on revendique dans l'espace public n'est pas aisément applicable dans la sphère privée. Le corps est le lieu où peut se transmettre et s'incarner une série de réflexes genrés. Il y a donc de

9 Éric Macé, « Le piège de la "cause des femmes" », novembre 2003. Voir : http://Imsi.net. 
fortes chances que cette femme entretienne une dissonance cognitive avec elle-même mais influera positivement sur le conflit social.

Cette femme peut aussi souhaiter (re)qualifier son identité féminine. Elle conservera alors la partie d'elle-même dominée (ses comportements socialement induits qui l'amènent à reproduire un schéma hérité) mais vivra de manière " réconciliée ». Elle dépassera, dans ce cas, la dissonance cognitive mais reproduira le conflit social. Ainsi, il peut s'agir, alors, de transformer son propre rapport à soi : soit en disqualifiant chez soi les comportements genrés reproduits inconsciemment, soit, en se réconciliant avec son identité féminine, soit en dépasser l'idée même de jugements genrés.

\section{Femme et bricolage identitaire}

Catherine Thomas, dans son analyse "Accoucher naturellement : un choix écolo ? ", nous montre que certaines femmes désirent accoucher sans lourde prise en charge médicalisée, en vertu d'un respect pour le processus physiologique de la naissance et sans pour autant que celles-ci soient " essentialistes ». L'auteure nous montre qu'un autre mouvement « se revendiquant également féministe mais d'une tout autre manière, par une approche plus naturaliste, offre un sens communicatif à la douleur allant jusqu'à considérer déshumanisant de supprimer la douleur et avec elle le sens qu'elle peut porter dans le processus de la naissance. » « La médecine gère, mesure, traite, 'guérit', annihile la douleur haïe. »

Ainsi, on peut imaginer une femme aspirer à un accouchement sans accompagnement médicalisé (et en ce sens, « naturel ») sans pour autant souhaiter adopter tous les clichés de LA (la seule, son essence) femme, dans son couple. À l'inverse, une féministe constructiviste qui s'assurerait que toutes les tâches et les rôles sont partagés équitablement dans son couple pourrait souhaiter une assistance médicale poussée lors de son accouchement. Chaque femme opère donc un bricolage entre essentialisme et constructivisme.

Ce bricolage lui est d'ailleurs probablement nécessaire. Chaque prise de position, chaque choix, engagements dépend d'un héritage transmis et de déterminismes sociaux. En ce sens, aucune femme ne s'appartient totalement et toutes les femmes sont en prise avec un imaginaire social ou un inconscient collectif qui véhicule une série de clichés féminins (pour l'Occident ce serait lapidairement, compassion, don de soi, écoute, gentillesse, séduction, manipulation). Son corps, en étant soumis à un héritage familial et une série de pressions sociales, est le jeu d'incorporations. Comme Foucault l'avait fait remarquer, le corps est aussi le lieu du politique.

\section{Conclusion : constructivisme, désir et plaisir}

Ainsi, le problème que pose le constructivisme est celui du plaisir et du désir que la femme peut connaître à adopter des comportements genrés. Si le genre féminin est très 
certainement une construction sociale, cela n'empêche qu'une femme qui naît et qui vit dans un monde où on lui apprend à être émotive, sensible et attentive (par exemple) ne pourra pas se départir d'un désir de ce type ${ }^{10}$. La liberté pourrait devenir, dans ce cas et comme dans l'existentialisme sartrien, la capacité d'accepter une partie de ses déterminismes. Autrement dit, la capacité à consentir à son destin pour atteindre une jouissance. Si tel est le cas, une femme qui se vivrait de manière harmonieuse, réconciliée, serait alors une femme qui accepterait d'être en prise (plutôt qu'en proie) avec ses arrières fonds, malgré elle.

C’est la grande frontière qui sépare les écologistes-politiques et les deep-écologiste (que l'on pourrait qualifier d'écologistes orientées vers l'expérience du quotidien, plus proche de la sphère autonomiste). Les deep-écologistes, qui prennent en compte la nature de manière maximale, préfèreront réinvestir positivement leur nature identitaire que de s'engager dans un conflit social. À l'inverse, les écologistes «moins profonds » préfèreront la voie politique ou le conflit (social) comme levier de changement social à une « harmonisation » des tensions.

En effet, ce qui distingue les écologistes « militants » des écologistes « profonds » est le niveau de changement auquel elles vont s'atteler. Alors que les militantes chercheront à influer sur la structure et les mécanismes de la reproduction sociale, les écologistes « profondes » tenteront de s'aménager à espace de confort, d'harmoniser les différentes parties d'elles-mêmes pour coexister avec cet état de fait.

De manière caricaturale, les deep-ecologistes, proches de la doctrine essentialiste, prendront du plaisir dans les déterminismes sociaux dont elles sont l'objet et creusent une voie où, dans leur univers de pensée, la culture devient nature.

La posture d'engagement idéale serait probablement celle qui arrive à faire cohabiter un rapport à soi réconcilié sans que pour autant l'horizon du combat social ne soit évanoui.

\section{Bibliographie}

Deleuze G. (1994), « Désir et plaisir », Magazine littéraire n 325, octobre 1994.

de Beauvoir S. (1976), Le Deuxième Sexe, tome II, Paris : Gallimard.

Butler J. (2006), Défaire le genre, Paris : Éditions Amsterdam.

MacéÉ. (2003), « Le piège de la "cause des femmes" (Troisième partie) », novembre 2003. Voir : http://lmsi.net

\footnotetext{
${ }^{10}$ Un livre qui va dans ce sens est « Ethique de la sollicitude ». Ce livre apporte une réflexion autour de la valorisation du care, http://www.scienceshumaines.com/l-ethique-de-la-sollicitude_fr_15029.html
} 